\title{
A Retrospective Study on Clinical and Biochemical Profile of Neuroleptospirosis
}

\author{
Ajitha Kesi Chellappan¹, Regi David Sam², Padmakumar Rajashekharan Pillai ${ }^{3}$, Ajith Syamala Narayanan ${ }^{4}$ \\ 1, 2, 3, 4 Department of General Medicine, Government Medical College, Thiruvananthapuram, Kerala, India.
}

\section{ABSTRACT}

\section{BACKGROUND}

Leptospirosis is a zoonotic disease usually reported from South India. The clinical spectrum may range from asymptomatic infection, subclinical to a fatal hepatorenal syndrome. Most physicians are sensitized to diagnose leptospirosis, sometimes the diagnosis is missed, especially when associated with neurological manifestations. About 10-15 percent of cases can have neurological manifestations. These patients are referred to a tertiary care hospital due to poor therapeutic response. Since early management can be lifesaving, this study was carried out to evaluate the laboratory profile and clinical features in patients with neuroleptospirosis in a tertiary care centre in South India.

\section{METHODS}

This retrospective single centre study was conducted at Government Medical College, Thiruvananthapuram, Kerala. Neuroleptospirosis was diagnosed by clinical and laboratory findings of hepatorenal syndrome, and anti leptospira antibody detection by ELISA.

\section{RESULTS}

Total of 160 patients with leptospirosis were admitted during the period of study. 31 patients were diagnosed clinically as neuroleptospirosis. Out of 31 patients 21 $(67.7 \%)$ were males and $10(32.3 \%)$ were females. The mean age was $56.9 \pm 14.61$ years. (M: F 2:1, range 18-68 years,). The chief manifestations were fever in 29 (93.5 $\%)$, myalgia in 23(74.2\%), headache in 17 (54\%), jaundice in 14 (45\%), and conjunctival suffusion in 9 patients (29\%). Two patients (6.4\%) had altered sensorium and neck stiffness in 23 patients (74.19\%). Most common neurological manifestation was aseptic meningitis (87) followed by encephalitis (19.4\%), seizures in $9.7 \%$, mean WBC count was $17297.10 \pm 8948.413$ cells/microliter. Mean platelet count at admission was $88258.06 \pm 108727.478$ cell $/$ microliter. Mean serum creatinine was $3.9 \pm 2.3 \mathrm{mg} / \mathrm{dl}$. Seven \pm patients $(22.58 \%)$ succumbed to death. Mean age of patients who died were $50.63 \pm 11.94$ years $(p<0.084)$, mortality was more common in females $(50 \%)(p<0.074)$. Irrespective of age and sex, WBC count at admission $>18,800$ cell/ $\mu \mathrm{L}$ is associated with increased mortality $(p+0.032)$.

\section{CONCLUSIONS}

Early recognition of neuroleptospirosis in patients with hepatorenal dysfunction and altered sensorium is critical in the treatment of patients. Female gender and elevated WBC count at admission indicates poor prognosis.
Corresponding Author: Dr. Regi David Sam, Associate Professor, Glory Bhavan Mariyapuram P.O., Trivandrum-695122, Kerala, India. E-mail: regidavid1981@gmail.com

DOI: $10.14260 / j e m d s / 2022 / 12$

How to Cite This Article: Chellappan AK, Sam RD, Pillai PR, et al. A retrospective study on clinical and biochemical profile of neuroleptospirosis. $J$ Evolution Med Dent Sci 2022;11(01):61-65, DOI: $10.14260 /$ jemds/2022/12

Submission 06-01-2022, Peer Review 10-01-2022, Acceptance 20-01-2022, Published 25-01-2022.

Copyright (C) 2022 Ajitha Kesi Chellappan et al. This is an open access article distributed under Creative Commons Attribution License [Attribution 4.0 International (CC BY 4.0)]

\section{KEY WORDS}

Neuroleptospirosis, Prognosis, Aseptic Meningitis. 


\section{BACKGROUND}

Leptospirosis is a zoonotic disease commonly reported from south India. The spirochetes that cause leptospirosis are transmitted after direct contact with the urine, blood, or tissue from infected rodents. ${ }^{1}$ After an incubation period of one to two weeks, leptospirosis manifests as a biphasic illness consisting of an initial leptospiraemic phase lasting three to seven days followed by an immune phase lasting four to 30 days. ${ }^{2,3}$ The more severe form of disease with severe liver and kidney involvement associated with haemorrhage is known as Weil's disease. Neurological involvement in leptospirosis is otherwise referred to as neuroleptospirosis. Salient features of the organ involvements are described below. ${ }^{3}$ Mild to severe jaundice is the most important hepatic manifestation. Renal involvement is usually present in leptospirosis. Acute tubular necrosis (ATN) and interstitial nephritis are the usual renal manifestations. Pulmonary involvement: mild illness may manifest with occasional cough, blood-tinged sputum and chest discomfort. In severe cases, patients can have severe cough, haemoptysis, progressive breathlessness which may lead to respiratory failure and death. Pulmonary haemorrhage and acute severe respiratory distress can occur in leptospirosis which can lead to death. Death occurs within hours to two days. Cardiovascular system involvement can present with the following features like cardiogenic shock. Patients with shock can develop severe hypotension, cold clammy extremities, and tachycardia. Echocardiography usually reveals normal left ventricular systolic function since hypotension is either due to dehydration or peripheral vasodilatation. In arrhythmias patient presents with palpitations, syncope and irregular pulse. ${ }^{3}$ Central nervous system involvement in leptospirosis (neuroleptospirosis) commonly present as meningitis. ${ }^{4,5,6}$ Headache may be the only manifestation or irritability, restlessness, seizures and coma can occur.1,7 Encephalitis, focal deficits, spasticity, paralysis, nystagmus, peripheral neuropathies, nerve palsies, radiculitis, myelitis all have been reported. Differential diagnosis: Falciparum malaria, Dengue fever, Dengue haemorrhagic fever, scrub typhus, typhoid and viral hepatitis closely resemble leptospirosis and are prevalent in areas reporting leptospirosis. Other conditions to be differentiated include viral pneumonia, viral hepatitis, alcoholic hepatitis, acute encephalitis syndrome and pyelonephritis. ${ }^{8}$ Possibility of coinfections should be kept in mind. Recommended case definition for the management of cases of leptospirosisis are 3,9 suspected acute febrile illness with headache, myalgia and prostration associated with a history of exposure to infected animals or an environment contaminated with animal urine with one or more of the following: calf muscle tenderness, conjunctival suffusion, anuria or oliguria and/or proteinuria, jaundice, haemorrhagic manifestations (intestines, lung) meningeal irritation, nausea, vomiting, abdominal pain, and diarrhoea. Probable: Suspected case with positive presumptive laboratory diagnosis. Confirmed: Suspect/probable case with confirmatory laboratory test. (Note: The classification of suspected, probable and confirmed does not in any way explain the severity and that has to be assessed based on the severity and rapidity of organ involvement). Epidemics may be associated with periods of flooding. Cases in developed countries are related more to recreational activities such as swimming. ${ }^{2}$ Cases of leptospirosis are underreported, with 1500-2000 cases being reported globally every year. Though physicians and internists in tropical countries are sensitized to diagnose a patient with characteristic clinical features, sometimes the diagnosis is missed because of atypical presentation, especially when associated with neurological manifestations. Neurological manifestations seen in about 10-15 percent of cases, remain unrecognized and diverse. ${ }^{7}$ This subgroup of patients is empirically treated for cerebral malaria, dengue fever, tuberculous meningitis, hepatic encephalopathy, viral encephalitis, etc., 1,6,10 based on seasonal prevalence, endemicity of the infective agent and clinical bias. They are referred to a tertiary care hospital, following poor therapeutic response, often in a moribund state. ${ }^{11}$ Hence, it is very important to be aware of these uncommon manifestations of leptospirosis, especially in patients with neurological deficits. Early recognition and management of such cases will be lifesaving.

This study was conducted to evaluate the clinical features and laboratory profile of patients presenting with neurological manifestations, following leptospirosis infection (neuroleptospirosis) in a tertiary care hospital in South India to analyse the clinical outcome and the associated prognostic indicators.

\section{METHODS}

To conduct this retrospective single centre study, we recruited patients from January 2019 to January 2020 at Government Medical College Thiruvananthapuram, Kerala, India. This is a hospital mainly for adult patients (i.e.; age $\geq 12$ years) specialising in managing patients with infectious diseases. All patients who were diagnosed as having leptospirosis according to WHO interim guidance were enrolled in this study. The study was approved by Government Medical College Hospital Ethics Committee. This census type of cross-sectional study was carried out after reviewing the consecutive case files of 150 patients who were admitted with leptospirosis during the study period, under internal medicine department. Trivandrum is located in a temperate zone, with significant rodent population in the environment. We obtained epidemiological, demographic and, clinical history (i.e.; history of patient symptoms and signs referable to nervous system involvement). Of the 150 patients, 31 had evidence of neuroleptospirosis. Clinical, laboratory, management, and outcome data from patients were recorded from medical records. If data were missing from the records or clarification was needed, we obtained data by direct communication with attending doctors and other health-care providers. All data were checked by two physicians. Laboratory confirmation of leptospiral infection was done by IgM ELISA. Detection of IgM antibodies to leptospira species was determined using a commercially available leptospira IgM ELISA (Panbio Pvt, Ltd). Patients who had same neurological manifestations but were diagnosed to have cerebral malaria, enteric encephalopathy, tuberculous meningitis, viral encephalitis, dengue fever with neurological deficits, or septic encephalopathy were excluded from the study. Blood parameters included complete hemogram and platelet count. Reports of biochemical investigations like, renal function tests, liver function test, 
serum amylase, lipase, troponin serum electrolytes and imaging modalities like chest $\mathrm{x}$ rays, ultrasound, CT head were taken for analysis.

\section{Statistical Analysis}

The collected data was analysed using SPSS version 17 software (IBM SPSS Statistics). Categorical data were analysed and presented as percentages. Mean and standard deviation were used to express quantitative data. The relationship between mild and severe cases was evaluated using logistic regression, using severe as the dependent or outcome variable. Variables which had significant finding in univariate analysis for the severe cases were further analysed by multivariable logistic regression test to predict the independent variable for severity. Only p-value less than 0.05 with a corresponding 95\% confidence interval (CI) of more than one was taken as a significant result.

\section{RESULTS}

\begin{tabular}{|cc|}
\hline Symptoms & Number of Patients (\%) \\
Fever & $2993.5 \%$ \\
Jaundice & $1445.2 \%$ \\
Bilateral conjunctival suffusion & $929 \%$ \\
Meningism & $2787 \%$ \\
Myalgia & $23(74.2 \%)$ \\
Headache & $1754.8 \%$ \\
Hypotension & $825.8 \%$ \\
Albuminuria/ Nitrogen retention & $2271 \%$ \\
Cough & $1238 \%$ \\
Anicteric patients & $1754.8 \%$ \\
Fever high grade & $2890.3 \%$ \\
\hline Table 1. Clinical Profile of Neuroleptospirosis \\
\hline
\end{tabular}

\begin{tabular}{|ccc|}
\hline Complications & Number of Patients Affected & $\mathbf{\%}$ \\
Neuroleptospirosis & 31 & \\
Aseptic meningitis & 27 & $87.1 \%$ \\
Encephalitis & 6 & $19.4 \%$ \\
Myeloradiculopathy & 1 & $3.2 \%$ \\
Myelopathy & 1 & $3.2 \%$ \\
GBS & 1 & $3.2 \%$ \\
Encephalopathy & 1 & $3.2 \%$ \\
Neuro myelitis optica & 1 & $3.2 \%$ \\
Seizures & 3 & $9.7 \%$ \\
Myoclonic jerks & 1 & $3.2 \%$ \\
Acute kidney injury SCr >/1.5 mg/dl & 23 & $74.2 \%$ \\
AKI SCr $\geq 3$ mg/dl & 13 & $41.9 \%$ \\
AKI requiring haemodialysis & 9 & $29 \%$ \\
AKI requiring haemodialysis cycle $\geq 2$ & 6 & $19.4 \%$ \\
Hepatorenal & 22 & $71 \%$ \\
Platelet count <25,000(cmm) & 7 & $22.6 \%$ \\
Bleeding diathesis & 3 & $9.7 \%$ \\
Acute lung injury & 3 & $9.7 \%$ \\
Acute lung injury requiring ventilator & 2 & $6.5 \%$ \\
support & 6 & $19.4 \%$ \\
Bronchopneumonia & 4 & $23.5 \%$ \\
Myocarditis (echo proven) & 9 & $29 \%$ \\
Pancreatitis & 3 & $9.7 \%$ \\
Acalculous cholecystitis & 8 & $25.8 \%$ \\
Hypotension & & \\
\hline Table 2. Complications of Neuroleptospirosis & \\
\hline & & \\
\hline
\end{tabular}

Total of 160 patients who were diagnosed with leptospirosis were admitted during the period of study. 31 patients were diagnosed clinically as neuro leptospirosis. Out of 31patients, 21 (67.7\%) were males and 10 (32.3\%) were females. The mean age was $56.9 \pm 14.61$ years. (Male to female ratio 2:1, age ranging from eighteen to sixty-eight years). Fever with chills and rigors in 29 (93.5 \%), myalgia in 23 (74.2\%), headache in 17 (54\%), and jaundice in 14 (45 $\%)$ were the chief manifestations.
Two patients (6.4\%) had altered sensorium, and neck stiffness was elicited in 23 patients (74.19\%). Conjunctival suffusion (with haemorrhage or without) was seen in 9 patients (29\%), and mild splenomegaly in 1 (3.2\%).

Most common neurological manifestation was aseptic meningitis ( $87 \%$ ) followed by encephalitis (19.4\%), seizures in $9.7 \%$, while myelopathy myeloradiculopathy, GBS and neuromyelitis optica contributed to one case each. Papilloedema was observed in two. CT scan of head was normal in 30 of 31 (96\%), while one had diffuse cerebral oedema. Pancreatitis was observed in 9 [29 \%]. Three patients had bronchopneumonia, of whom two patients required ventilator support. Splenomegaly was found in one patient (3.2\%), hepatomegaly was noted in one patient (3.2 $\%)$. Mean serum sodium was $131.48 \pm 7.3$ milliequalents per litre, mean serum potassium was $4.3 \pm 0.88$ millieqaulents per litre. Hypotension was noted in eight patients (25.8). Myocarditis was noted in 4 (23.5\%) Mean WBC count was $17297.10 \pm 8948.413$ cells/microliter. Mean platelet count at admission was $88258.06 \pm 108727.478$ cell/microliter. Mean blood urea was $113,87 \pm 92.326 \mathrm{mg} / \mathrm{dl}$, mean serum creatinine was $3.9 \pm 2.3 \mathrm{mg} / \mathrm{dl}$. of whom nine patients underwent haemodialysis. Mean serum bilirubin was $7.2 \pm$ $6.39 \mathrm{mg} / \mathrm{dl}$, mean serum SGOT was $129.10 \pm 125.41 \mathrm{U} / \mathrm{L}$. Mean serum SGPT $120 \pm 170.47$ U/l. 7 patients (22.58\%) succumbed to death. Mean age of patients who died was $50.63 \pm 11.94$ years $(p<0.084)$, eight patients succumbed to death (25.8\%). Mortality was more common in females (50\%) compared to males (14.3\%), $(\mathrm{p}<0.074)$. Irrespective of age and sex, WBC count at admission $>18,800$ cell/microliter is associated with increased mortality (ROCarea under curve=0.758: Log rank test, $p+0.032$ ). Socioeconomic factors and clinical features like headache fever, neck stiffness, conjunctival suffusion, meningism, myalgia, jaundice did not affect the mortality. Mean WBC count of patients who died was $21320 \pm 11256.023$ (p value 0.005). Platelet count did not affect the mortality. Liver function test like SGOT, SGPT, serum bilirubin, alkaline phosphatase did not affect the mortality. Blood urea at admission (mean $165.50 \pm 110.84 \mathrm{mg} / \mathrm{dl}$ ) was significantly high in patients who died. Mortality was high in patients who had hypotension (6 patients, $\mathrm{p}$ value by Fischer exact 0.0007 ]. None of the patients had bleeding complications and coagulation profile was normal in all. In our study, type of antibiotics used did not affect the mortality.

Variables selected for multivariate analysis were age, sex, WBC count at admission, blood urea maximum, and blood pressure. Male sex is an independent factor favouring survival after adjusting for age and WBC Maximum $(\mathrm{p}=$ 0.037). Irrespective of age and sex, WBC maximum is an independent predictor of survival $(\mathrm{p}$ value $=0.047$ )

\begin{tabular}{|c|c|c|c|c|c|c|}
\hline & \multirow{2}{*}{ Wald } & \multirow{2}{*}{ Sig. } & \multirow{2}{*}{$\operatorname{Exp}(B)$} & \multicolumn{2}{|c|}{$95 \%$ C. I. for $\operatorname{Exp}(B)$} \\
\hline & & & & & Lower & Upper \\
\hline \multirow{4}{*}{$\begin{array}{c}\text { Step } \\
1^{\mathrm{a}}\end{array}$} & Age & 1.145 & .285 & .956 & .881 & 1.038 \\
\hline & Sex M (1) F (1) & 4.342 & .037 & 19.910 & 1.195 & 331.832 \\
\hline & WBC Maximum & 3.963 & .047 & & & \\
\hline & Constant & 3.964 & .046 & 228.853 & & \\
\hline
\end{tabular}




\section{ROC Cure Analysis for WBC MAX}

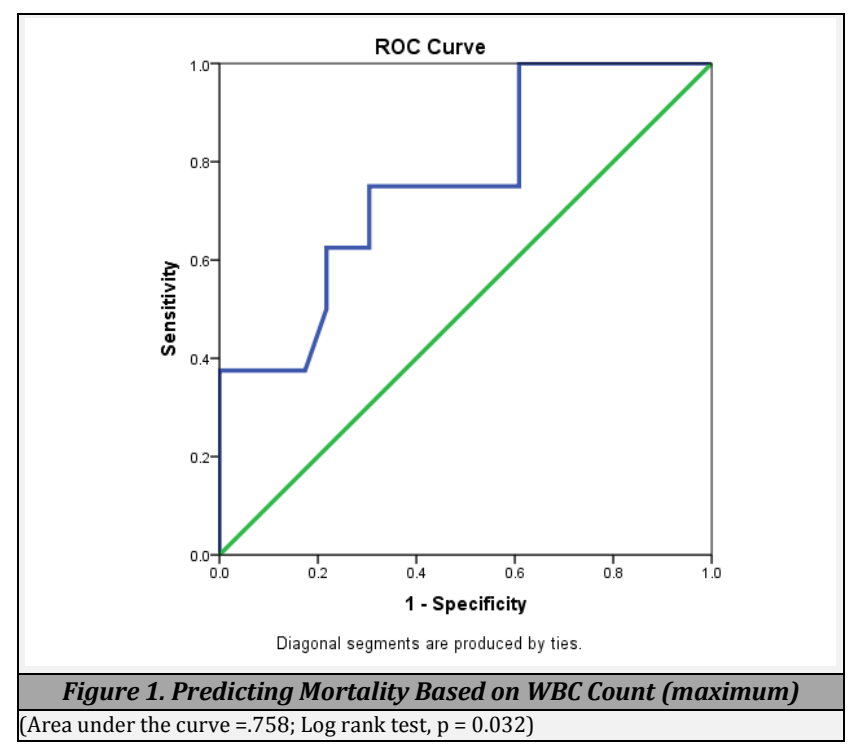

\begin{tabular}{|ccc|}
\hline $\begin{array}{c}\text { WBC Maximum [cells } / \boldsymbol{\mu L} \text { ] } \\
\text { Positive if Greater Than or Equal } \\
\text { to }^{\mathbf{a}}\end{array}$ & Sensitivity & Specificity \\
10000.00 & 1.000 & 0.261 \\
10500.00 & 1.000 & 0.304 \\
11000.00 & 1.000 & 0.348 \\
11700.00 & 1.000 & 0.391 \\
12200.00 & .875 & 0.391 \\
12390.00 & .750 & 0.391 \\
12490.00 & .750 & 0.435 \\
13000.00 & .750 & 0.478 \\
13700.00 & .750 & 0.522 \\
14250.00 & .750 & 0.565 \\
15500.00 & .750 & 0.609 \\
17400.00 & .750 & 0.652 \\
18800.00 & .750 & 0.696 \\
19235.00 & .625 & 0.696 \\
19335.00 & .625 \\
\hline \multicolumn{2}{|c|}{ Table 4. Coordinates of the Curve } \\
\hline
\end{tabular}

A cut off of 12200 cells/ $\mu \mathrm{L}$ has a sensitivity of $87.5 \%$ and specificity of $39.1 \%$. Raising the cut off to 12390 cells/ $\mu \mathrm{L}$ or 12490 cells/ $\mu$ had little effect of increasing the specificity. As per our data, the best trade-off between sensitivity and specificity was at a cut off of 18800 cells/ $\mu \mathrm{L}$ which had $75.0 \%$ sensitivity and $69.6 \%$ specificity.

\section{DISCUSSION}

The clinical manifestation and presentation of leptospirosis are broad and only specific when it becomes severe.3,12 This presents challenges to clinicians not only to make a correct diagnosis but also to give the best management and treatment to the patients to prevent progression to severe disease and mortality. Several studies have outlined the prognostic factors associated with severe and fatal leptospirosis ${ }^{11}$; however, the predictors could be different between geographical location depending on the sociodemography, type of occupation, prevailing leptospira species, serovars and strains. ${ }^{8}$

In the present study, most leptospirosis patients were males, and this might be due to exposure-related bias where males are more involved with outdoor activities and may have occupational-related risk exposure. ${ }^{11}$ Patients presented with typical manifestation of leptospirosis such as fever, headache, myalgia and gastrointestinal involvement such as nausea and abdominal pain. Age above 70 years together with organ dysfunction has been reported as a predictor for fatality in an earlier study from Malaysia, however ${ }^{2}$ it is undoubtedly evident that older age worsens the disease course, and the reason it was not significant for severity in our study, could be due to the low number of patients aged above 60 years.

Eight variables (mechanical ventilation, AKI, septic shock, creatinine level of $>1.13 \mathrm{mg} / \mathrm{dL}$, urea $>7 \mathrm{mmol} / \mathrm{L}$, ALT $>50$ IU, AST $>50 \mathrm{IU}$, and platelet $<150 \times 10^{9} / \mathrm{L}$ ) were not found to be associated with severe illness in this study. ${ }^{8}$ The independent predictor of severity included elevated total white blood cell count at admission in our study. One of the main limitations was since the clinical data were collected only on the day of admission, we were not able to associate the time period and change in values for the predictors with the severe illness. A recent study from Malaysia also showed the association of ALT with severe leptospirosis In addition to the increased ALT, this previous study also reported abnormal lung sounds, hepatomegaly, hypotension, leucocytosis and low haematocrit as the predictors for severe leptospirosis. ${ }^{11}$ We found an elevated WBC count more than 18800 per microlitre as one of the predictors of severe illness, this factor was suggested in the above study. ${ }^{2}$ This present study and two other studies in Malaysia showed lung involvement as one of the predictors of severity and mortality in leptospirosis. Leptospirosis patients with pulmonary haemorrhage was also reported in several case reports including travellers who visited Malaysia.2,10 These factors were found to be the independent factors for mortality in studies conducted in several other countries. Similar to the present investigation, studies from other countries have also reported the association of exaggerated AST response and higher mean of aspartate/alanine aminotransferase ratio (AAR) with severity and mortality in leptospirosis. ${ }^{12}$ These findings indicate that severe leptospirosis in Malaysia was characterized with multi-organs involvement. ${ }^{2}$ Progressive monitoring and measurements of these multi-factorial data even in patients presenting mild symptoms and with history of activities or exposure to potential source of leptospira is vital to prevent the development of severe disease. Since these data were obtained during admission, patients are advised to seek medical treatment early if they presented with mild symptoms after jungle or water activities, exposed to flood or if working in areas with a high risk of rat infestation as the transition of mild to severe disease to fatality occur rapidly.

The data obtained from the present study provides baseline information on the factors associated with severe leptospirosis and this needs to be further evaluated on a larger sample size covering different geographical locations across the globe. Acute kidney injury was the common non neurological complication followed by hepatorenal dysfunction and pancreatitis.

The prognosis of neuroleptospirosis is largely unknown. Most of the studies report mortality rates for systemic leptospirosis, varying from $5-15 \% .^{7}$ In the study by Singh et al. mortality rate was $24.1 \%$ and the major cause of mortality was pulmonary involvement. ${ }^{12}$ In the study of Health et al. ${ }^{1}$ which included patients with neurological and nonneurological manifestations, the mortality was $7 \% .{ }^{11}$ In this 
study $26 \%$ of patients succumbed to the infection, similar to the study by Mathew et al. ${ }^{7}$ This high mortality could be due to late referral and subsequent delay in management. Mathew et al. demonstrated that raised CSF protein concentration and altered sensorium at the time of admission were found to be responsible for poor prognosis. ${ }^{7}$ In the present study male sex is an independent factor favouring survival. Irrespective of the age and sex, WBC count (maximum) more than $18,800 \mathrm{cell} /$ microlitre was associated with increased mortality due to neuroleptospirosis. Increase in age, female sex, increase in blood urea, serum creatinine, and thrombocytopenia were more marked in the those who succumbed but they did not reach statistical significance, probably due to small sample size and hence needs further evaluation. Most common neurological manifestation was aseptic meningitis (87\%) followed by encephalitis (19\%), seizures were rare $(9 \%)$. The exact mechanism of seizures in neuroleptospirosis is unknown. The seizures could be secondary to diffuse encephalitis following leptospiral infection or due to metabolic dysfunction related to hepatorenal syndrome. Except for altered sensorium and neck stiffness, neurological examination did not reveal any focal neurological deficits in majority of the patients. In the study by Panicker et al. paraparesis was the initial presentation in 17 patients. ${ }^{4}$ Three patients had lower motor neuron paraparesis. Myelopathy was seen in seven patient's, myeloradiculopathy in seven and Gullian Barre syndrome like presentation in three. ${ }^{4}$ Similarly in the present study, myelopathy, myeloradiculopathy, Gullian Barre syndrome and neuromyelitis optica contributed to one case each. CT scan was normal in all patients with neurological dysfunction. Mathew et al. in his study suggested that one needs to consider neuroleptospirosis in the differential diagnosis, whenever a diagnosis of viral encephalitis is made, especially in endemic areas with seasonal prevalence. ${ }^{7}$ The present study demonstrates the same conclusion of neuroleptospirosis. ${ }^{7}$ An early diagnosis of neuroleptospirosis is mandatory as effective and specific treatment is available and neurological sequelae are unusual. The role of steroids in the treatment of leptospirosis during the immune phase is controversial. The present study did not show any significant difference in mortality among patients receiving steroids. The role of steroids in patients with neuroleptospirosis is not known and needs to be evaluated in future studies.

\section{CONCLUSIONS}

In conclusion, neuroleptospirosis is an important diagnosis which should be considered in the differential diagnosis of all patients presenting with hepatorenal dysfunction and altered sensorium especially in areas where leptospirosis is endemic during monsoon season. High index of suspension is needed for timely diagnosis and early initiation of treatment. Male sex is an independent factor favouring survival and WBC count more than 18,800 per microliter is associated with poor prognosis

Early recognition of neuroleptospirosis is critical in the treatment of patients in endemic areas. Female gender and elevated WBC count at admission indicates poor prognosis.

Data sharing statement provided by the authors is available with the full text of this article at jemds.com.

Financial or other competing interests: None.

Disclosure forms provided by the authors are available with the full text of this article at jemds.com.

\section{REFERENCES}

[1] Heath CW, Alexander AD, Galton MM. Leptospirosis in the United States. Analysis of 483 cases in man, 19491961. New Engl J Med 1965;273(17):915-22.

[2] Neela VK, Azhari NN, Joseph N, et al. An outbreak of leptospirosis among reserve military recruits, Hulu Perdik, Malaysia. Eur J Clin Microbiol Infect Dis 2019;38(3):523-8.

[3] Speelman P. Leptospirosis. In: Fauci AS, Braunwald E, Isselbacher KJ, et al, eds. Harrison's principles of internal medicine, $14^{\text {th }}$ edn. New York: Mcgraw-Hill Company 1998:1036-8.

[4] Panicker JN, Mammachan R, Jayakumar RV. Primary neuroleptospirosis. Postgrad Med J 2001;77(911):58990.

[5] Gancheva GI. Involvement of central nervous system in leptospirosis. J Biomed 2009;2(2):109-14.

[6] Beeson PB, Hankey DD. Leptospiral meningitis. AMA Arch Intern Med 1952;89(4):575-83.

[7] Mathew T, Satishchandra P, Mahadevan A, et al. Neuroleptospirosis-revisited: experience from a tertiary care neurological centre from south India. Indian J Med Res 2006;124(2):155-62.

[8] Cacciapuoti B, Ciceroni L, Maffei C, et al. A waterborne outbreak of leptospirosis. Am J Epidemiol 1987;126(3):535-45.

[9] WHO. Report of the second meeting of the leptospirosis burden epidemiology reference group. Geneva: World Health Organization 2011.

[10] Shivakumar S, Shareek PS. Diagnosis of leptospirosis utilizing modified Faine's criteria. J Assoc Physicians India 2004;52:678-9.

[11] Philip N, Than LTL, Shah AM, et al. Predictors of severe leptospirosis: a multicentre observational study from Central Malaysia. BMC Infect Dis 2021;21(1):1081.

[12] Singh SS, Vijayachari P, Sinha A, et al. Clinicoepidemiological study of hospitalized cases of severe leptospirosis. Indian J Med Res 1999;109:94-9. 